\title{
Enhanced dispersion interaction between quasi-one-dimensional conducting collinear structures
}

\author{
Angela White ${ }^{1,2, *}$ and John F. Dobson ${ }^{1, \dagger}$ \\ ${ }^{1}$ Nanoscale Science and Technology Centre, Griffith University, Nathan, Queensland 4111, Australia \\ ${ }^{2}$ Centre for Gravitational Physics, Department of Physics, Faculty of Science, The Australian National University, \\ Canberra, Australian Capital Territory 0200, Australia \\ (Received 27 September 2006; revised manuscript received 5 November 2007; published 29 February 2008)
}

\begin{abstract}
Recent investigations have highlighted the failure of a sum of $R^{-6}$ terms to represent the dispersion interaction in parallel metallic, anisotropic, linear, or planar nanostructures [J. F. Dobson, A. White, and A. Rubio, Phys. Rev. Lett. 96, 073201 (2006), and references therein]. By applying a simple coupled-plasmon approach and using electron hydrodynamics, we numerically evaluate the dispersion (noncontact van der Waals) interaction between two conducting wires in a collinear pointing configuration. This case is compared to that of two insulating wires in an identical geometry, where the dispersion interaction is modeled both within a pairwise summation framework and by adding a pinning potential to our theory leading to a standard oscillator-type model of insulating dielectric behavior. Our results provide a further example of enhanced dispersion interaction between two conducting nanosystems compared to the case of two insulating ones. Unlike our previous work, this calculation explores a region of relatively close coupling where, although the electronic clouds do not overlap, we are still far from the asymptotic region where a single power law describes the dispersion energy. We find that strong differences in dispersion attraction between metallic and semiconducting or insulating cases persist into this nonasymptotic region. While our theory will need to be supplemented with additional short-ranged terms when the electronic clouds overlap, it does not suffer from the short-distance divergence exhibited by purely asymptotic theories and gives a natural saturation of the dispersion energy as the wires come into contact.
\end{abstract}

DOI: 10.1103/PhysRevB.77.075436

PACS number(s): 81.07.De, 61.46.Fg, 34.20.Cf, 73.22.-f

\section{INTRODUCTION}

The dispersion interaction constitutes the outer, attractive part of the van der Waals (vdW) force (in the nonretarded regime, for charge-neutral, nonpolar species). It can be explained via the interaction between small instantaneous dipoles arising due to a mutual dynamic polarization of the electron clouds (see, e.g., Ref. 1). These attractive forces, although weaker in magnitude than ionic or covalent bonds between atoms or molecules, are ubiquitous across nature and can play a central role in soft matter and biophysical systems. Examples range from protein folding ${ }^{2-4}$ to the adhesive properties of gecko feet. ${ }^{5,6}$ While the present work cannot describe the contact regime where the vdW force is strongest, it does suggest that noncontacting regions may contribute more than previously suspected to the energetics, in cases where pi-conjugation, for example, may lead to near-metallic behavior in low-dimensional structures.

Dispersion forces also play an important role in the rapidly progressing area of nanoscience. Nanotubes are quasione-dimensional structures, with electronic properties determined by their composition. The metallic or semiconducting behavior depends on the helicity of carbon nanotubes. Nanotubes composed of boron nitride are primarily semiconducting, with a wide band gap and stable electronic properties. ${ }^{7,8}$ Armchair $(n, n)$ carbon nanotubes are metallic. The $\mathrm{vdW}$ attraction facilitates the self-assembly of single-walled nanotubes into bundles or ropes ${ }^{9}$ and being the primary intertube attraction is important in a description of the cohesive energetics of nanotube bundles. ${ }^{10}$

Recently, the differing asymptotic behavior of $\mathrm{vdW}$ interactions for conducting systems, compared to insulating sys- tems with identical geometry, has been displayed for various systems. ${ }^{111-13}$ The simplest standard techniques applied in computing the vdW interaction are based on the pairwise summation of all $R^{-6}$ contributions ${ }^{14}$ between microscopic elements separated by distance $R$ and so inherently assume local properties of the two materials. More sophisticated techniques ${ }^{15,16}$ when coupled with the usual assumption of a local bulk-like dielectric function within the boundaries of the constituents still lead to an interaction asymptotically equivalent to a sum of $R^{-6}$ contributions. Recently, progress has been made in formalisms that describe both the contact region of electron cloud overlap, as well as a dispersion contribution at large distances. ${ }^{17-25}$ However, these theories predict the usual asymptotic $\Sigma R^{-6}$ behavior and so could still be improved with respect to the description of low-dimensional systems with a small or zero electronic energy gap.

For a determination of the $\mathrm{vdW}$ interaction between highly anisotropic systems of metallic nature, a pairwise summation method fails to incorporate the long wavelength, incompletely screened electron density fluctuations. These lead to unexpected power laws for the vdW interaction as a function of separation. ${ }^{12}$ The effects of this physics appear in calculations on parallel one-dimensional (1D) electron gases, ${ }^{26}$ on two-dimensional electron gases, ${ }^{27-29}$ and in graphene sheets. ${ }^{12}$ More recently, this has been highlighted as a more general phenomenon. ${ }^{12}$ The greatest underestimation of the $\mathrm{vdW}$ interaction occurs between distant parallel quasi-one-dimensional conductors. ${ }^{12,26,30}$

The coupled-plasmon approach addresses the shortfalls of the pairwise summation technique, incorporating polarization between multiplets of atoms in the system and also electron movement within the wires. In modeling the electronic 
motions as coupled plasmons, large electron displacement across many atoms in a system is allowed to occur, providing a less constrained representation of electron movement. Plasmon frequencies can be determined by application of electron hydrodynamics, and the $\mathrm{vdW}$ dispersion energy then corresponds to the separation dependent part of the sum of zero-point plasmon energies. This approach has been employed previously in the determination of the vdW interaction between systems of thin parallel metallic plates and also thin parallel metallic wires. The sum of plasmon energies is also known to provide an approximation to the full correlation energy in the random phase approximation (RPA) (see, e.g., Ref. 13). A full RPA energy calculation would thus provide a natural and seamless extension to the present method, valid at all separations including the regime of full electronic cloud overlap.

For two thin parallel metallic plates of infinite area, separated by a distance greater than the thickness of the plates, Böstrom and Sernelius ${ }^{29}$ and Dobson and Wang ${ }^{28}$ have applied the coupled-plasmon approach to obtain a dispersion energy of dependence $D^{-5 / 2}$ on the separation distance $D$ of the metal slabs. This result is in agreement with the appropriate limit of microscopic random phase approximation calculations for a pair of two-dimensional electron gases performed by Sernelius and Björk. ${ }^{27}$ A pairwise summation analysis for this system of thin parallel metallic plates gives a $D^{-4}$ dependence of the vdW attraction, notably smaller at large separation than the $D^{-5 / 2}$ dependence derived by applying the coupled-plasmon approach. An equally unconventional result was obtained for the interaction between graphene planes. ${ }^{11,12}$

Another example system in which the pairwise summation analysis was shown to underestimate the dispersion interaction for conductors is that of two parallel wires of infinite length. ${ }^{12,26,30,31}$ For a pair of parallel, conducting, infinitely long wires, the dispersion interaction, calculated by a standard sum of $R^{-6}$ contributions between microscopic elements separated by distance $R$, has a $D^{-5}$ dependence on the separation distance $D$ of the two wires. However, an analysis of the zero-point energy of the delocalized coupled 1D plasmon modes parallel to the long axis, modeled for wires of length $L \gg D$, finds the $\mathrm{vdW}$ interaction to have a

$$
D^{-2}\left[\ln (D / A)^{-3 / 2}\right]
$$

dependence on separation distance $D$. A denotes the smearing radius of a wire, representing the finite extent of the electronic wave function or electronic density fluctuation on the wire in the direction perpendicular to the long axis. The analysis assumes an electron mean free path greater than the separation distance of the wires, which can be satisfied by bismuth nanowires ${ }^{32}$ and conducting nanotubes. ${ }^{10,33}$ The $\mathrm{vdW}$ interaction [Eq. (1)] is almost 3 powers of $D$ greater than that obtained in a standard pairwise summation framework. This highlights the importance of including electron movement and screening from subsequent polarization of electron pairs in conducting systems. The occurrence of an enhanced dispersion interaction in metallic systems might have repercussions, particularly in nanotechnology. The above considerations suggest that metallic and nonmetallic

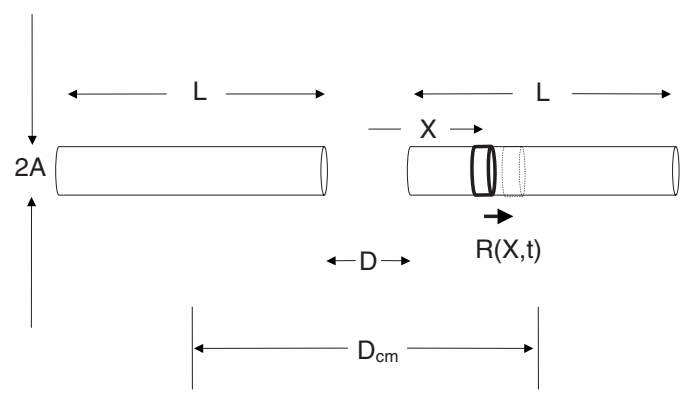

FIG. 1. Geometry for electron density perturbations on pointing wires.

nanotubes could experience different cohesive forces, for example, though analysis beyond the present methods would be needed to explore this possibility in the case of electron cloud overlap. In the present paper, we explore another case $^{31}$ where a sum of $R^{-6}$ contributions does not yield a correct description of the vdW interactions. We consider two linear structures ("wires"), each of length $L$, in a collinear "pointing" configuration as shown in Fig. 1. $D$ is the separation between the near ends of the wires, while $D_{c m}$ is the distance between the centers of the wires. $A$ is an effective radius of the wire, which we will discuss further in Sec. II.

Our motivation for looking at this case was threefold. First, there is intrinsic interest in the cohesive properties of nanostructures of all kinds, and the present work is particularly relevant to the interactions of carbon nanotubes, a technologically important case. Second, recent work ${ }^{34}$ has suggested that $\mathrm{vdW}$ forces may be involved in the surprisingly strong force that tends to make the tips of iron microwhiskers grow toward one another during fluidized-bed iron ore reduction processes. While it is not clear that the electron mean free path in these cases is sufficiently long to validate the assumptions of the present work, it is interesting that our approach can predict vdW forces between linear conducting systems in the appropriate pointing geometry that are enhanced compared with standard vdW theory. Third, a numerical investigation of wires of finite length allows us to look at a region of relatively close coupling where, although the electronic clouds are not permitted to overlap, we are still far from the truly asymptotic regime where a single power law describes the force. We find that the dispersion interaction between the wires shows strong differences between metallic and insulating cases even in this nonasymptotic regime, reinforcing asymptotic results ${ }^{11,12,26,27,29}$ already known in other geometries. We further find that the greater interaction at large distances in the metallic case does not imply a lesser interaction at small distances in the metallic case, contrary to a false expectation based on a single power law at all distances.

The paper is organized as follows. The analytic work will be presented in Sec. II, and Sec. III then outlines the numerics. Our Results and Discussion compare the dispersion interaction between wires in the pointing geometry for insulators and conductors and are presented in Sec. IV. The principal findings and future prospects are discussed in the final section. 


\section{ANALYTICS}

We evaluate the dispersion interaction between the two wires by the coupled-plasmon approach (see, e.g., Refs. 12-14). Plasmons are quantized versions of the wavelike collective motions of the electrons. The equilibrium and/or ground state of the wire is described by a constant electron number density per unit length, $n_{0}$. Plasma waves entail a perturbation $\delta n(X, t)$ to the number density, where we have defined a variable $X$ (see Fig. 1) to label positions along the wire.

Here, we neglect electronic radial and azimuthal motions, i.e., those perpendicular to the long axis. These are frozen out by quantum effects in atomically thin systems but can be present in other cases. We do not consider them here because, for the thin linear systems of interest here, they consist of spatially constrained electronic motions leading to conventional vdW force laws. By contrast, the unconstrained electronic motions along the wire will be shown below to lead to an unconventional, enhanced long-ranged vdW interaction that is the principal focus of attention here. For example, the azimuthal electronic motions have been considered by Rotkin and Hess ${ }^{35}$ in the case of two long parallel nanotubes. They considered only small separations between the tubes and found these modes to give a more rapidly decaying vdW interaction as a function of nanotube separation $D$, compared to the purely longitudinal modes investigated in Ref. 11. Thus, we expect that our neglect of the electronic motions perpendicular to the long axis will not affect the long-ranged forces that we are investigating here.

To describe the electronic motions along the wire, we use a conventional hydrodynamical picture, valid even for degenerate quantal electrons in the limit of long-wavelength perturbations. During the plasmon motion, the electron fluid element that was at position $X$ (see Fig. 1) in the unperturbed state of the wire (bold lines in Fig. 1) is displaced to position $X+R(X, t)$ (dotted lines in Fig. 1). In the simplest hydrodynamical model (correct to lowest order in the wave number $Q$ of the wave), the motion of the fluid element is described by Newton's second law for a free mass under the action of a mean potential energy function $\Phi(X, t)$ generated by the Coulomb interaction with other fluid elements: $M d^{2} R(X, t) / d t^{2}=-\partial \Phi(X, t) / d X=F$. Here, $M$ is the electron effective mass for motion along the wire.

An element of electron fluid at a point on any wire will experience a Coulomb potential due to electrons at every other point on that wire and also due to those at every point on the adjacent collinear wire. Considering plasmons in one dimension, evidently, the Fourier transform of the Coulomb potential does not converge. However, by recognizing that the one electron wave functions are of finite extent in the direction perpendicular to the wire, we can examine a symmetrically "smeared" version of the wire with finite smearing radius $A$. While we apply a radially smeared pair potential, expressed explicitly as

$$
\widetilde{\phi}\left(\left|X-X^{\prime}\right|\right)=\frac{e^{2}}{\left[\left(X-X^{\prime}\right)^{2}+A^{2}\right]^{1 / 2}}
$$

in our work to follow, any expression that saturates the Coulomb interaction for $|r|<A$ would give similar results for the long-wavelength fluctuation phenomena that drive the unusual effects to be explored here.

The force on an element of electron fluid at a point in time can be expressed using the radially smeared pair potential $\widetilde{\phi}$ as

$$
\begin{aligned}
M \frac{d^{2} R(X, t)}{d t^{2}}= & -\frac{\partial}{\partial X}\left(\int_{-D / 2-L}^{-D / 2} \delta n\left(X^{\prime}, t\right) \widetilde{\phi}\left(\left|X-X^{\prime}\right|\right) d X^{\prime}\right. \\
& \left.+\int_{D / 2}^{D / 2+L} \delta n\left(X^{\prime}, t\right) \widetilde{\phi}\left(\left|X-X^{\prime}\right|\right) d X^{\prime}\right) .
\end{aligned}
$$

This applies for either $D / 2 \leqslant X \leqslant D / 2+L$ or $-(D / 2+L) \leqslant X \leqslant-D / 2$. We apply a linearized form of the continuity equation,

$$
\delta n(X, t)=-\frac{\partial}{\partial X}\left[n_{o}(X) R(X, t)\right]
$$

to describe the perturbation to the electron number density in terms of the equilibrium number of electrons per unit length of wire, $n_{o}(X)$.

Plasmon movement within metallic wires is confined by requiring zero electron displacement at the wire ends. An alternative analysis, in which the zero boundary condition occurring at the wire ends is naturally inherent, could be obtained by applying a sine-basis decomposition. Note that different boundary conditions, such as allowing the electron gas to move out over the ends of the uniform positive background, which would then cause a restoring force, could be applied instead and possibly would provide a further alternative description of the plasmon modes.

We seek time-periodic separable solutions of Eq. (3) in the form

$$
R(X, t)=R(X) \exp (-i \Omega t) .
$$

The left-right symmetry evident in Fig. 1 requires that there exist even solutions for which $R(X)=R(-X)$ and also odd solutions for which $R(X)=-R(-X)$. We used this property and put Eqs. (5) and (4) into Eq. (3). Integrating by parts and using the explicit form of the smeared Coulomb potential (2), we obtained

$$
\begin{aligned}
\frac{M \Omega^{2} R(X)}{n_{0} e^{2}}= & \int_{D / 2}^{L+D / 2} R\left(X^{\prime}\right)\left(\frac{-2\left(X-X^{\prime}\right)^{2}+A^{2}}{\left[\left(X-X^{\prime}\right)^{2}+A^{2}\right]^{5 / 2}}\right. \\
& \left. \pm \frac{-2\left(X+X^{\prime}\right)^{2}+A^{2}}{\left[\left(X+X^{\prime}\right)^{2}+A^{2}\right]^{5 / 2}}\right) d X^{\prime} .
\end{aligned}
$$

Equation (6) holds for either $-(L+D / 2) \leqslant X \leqslant-D / 2$ or $D / 2 \leqslant X \leqslant L+D / 2$ and is an eigenvalue equation for the frequencies $\Omega_{i}$ of self-sustaining plasma oscillations (plasmons), corresponding to coupled charge density fluctuations on the two wires.

\section{A. Evaluation of the dispersion energy}

The dispersion energy $E_{v d W}$ is then the part of the total plasmon zero-point energy that depends on the separation $D$ between the wires. Thus, 


$$
E_{v d W}(D)=\frac{\hbar}{2} \sum_{j}\left[\Omega_{j}(D)-\Omega_{j}(D \rightarrow \infty)\right] .
$$

Application of numerical techniques is necessary to solve for the eigenfrequencies $\Omega_{i}$, as the broken translational symmetry of the collinear wire geometry with a gap prevents Fourier transformation being used to solve the integral equation (6) analytically in $k$ space.

\section{B. Incorporating internal pressure}

Although the eigenvalue problem (6) derived from the expression for the force felt on an element of electron fluid at a point could now be solved to give us odd and even solutions for the plasmon frequency, we extend this to incorporate the contribution to the force felt by an element of electron fluid from the internal pressure of the electron fluid. This electronic pressure is caused mainly by velocity deviation of electrons in a fluid element from the average velocity. It is the electron pressure term that distinguishes our formalism from a purely classical Newton II approach, and it introduces the quantal and Pauli-principle physics (electron degeneracy pressure) into the formalism. We expect it should give higher frequencies to the plasmon modes with rapid spatial variation. Without the pressure term, the theory correctly describes the response of a free electron gas in the limit of long wavelength, and it agrees with the corresponding limit of the quantal Lindhard response. This limit was adequate in Ref. 12 because there the treatment was limited to the asymptotic regime of distant interactions between infinitely long wires, where the long-wavelength fluctuations completely dominate. Here, we want to explore wires of finite length at finite separations. While the phenomena which we expose will still turn out to be due to long-wavelength fluctuations, a detailed treatment needs to account, for example, for the reduced polarizability of a short wire of length $L$ resulting from the requirement to excite wavelengths of $O\left(L^{-1}\right)$ in order to polarize its electron gas. For this reason, we introduce the plasma pressure in the simplest available model. In some regimes, it also aids in achieving numerical stability of the solutions.

The excess pressure due to a density perturbation $\delta \rho$ is usually modeled from an analysis of the free Fermi gas ${ }^{36-41}$ as $\delta P=M B^{2} \delta \rho$. Here, $\delta \rho$ is the three-dimensional density perturbation and $B$ is a velocity of the order of the Fermi velocity of the metal. We take $\rho=n A^{-2}$. The pressure can now be expressed in terms of the equilibrium pressure $P_{0}$, and the perturbation to the electron number density per unit length, $\delta n=\delta \rho A^{2}$, as $P=P_{0}+A^{-2} M B^{2} \delta n$. Now, $B$ is of order of the Fermi velocity of the metal composing a wire. The pressure force per electron, to be added to the right side of Eq. (3), is then

$$
F=-\frac{1}{\rho_{0}} \frac{d P}{d X}=M B^{2} \frac{d^{2} R(X, t)}{d X^{2}},
$$

where from the linearized continuity [Eq. (4)], the perturbation to the electron number density has again been expressed as a function of the equilibrium number density $n_{0}$ and the displacement $R$ of an electron fluid element.

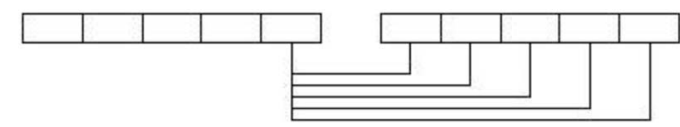

FIG. 2. Pairwise summation of an element of wire A with all corresponding elements on wire B.

\section{Dispersion interaction for insulating wires}

The prior analysis, applying a coupled-plasmon approach, accounts for conduction of electrons and so provides a more complete description in evaluating the vdW interaction between two conducting collinear wires. However, we would expect the pairwise summation method of individual $R^{-6}$ atomic contributions, which does not allow for electron movement along the wires, to remain an apt description of the dispersion interaction between insulating wires with a sufficiently large gap. We therefore applied two different modifications to the theory described above: Each is designed to explore the difference between metallic and nonmetallic wires.

(A) A modified pairwise additive approach was applied to calculate the dispersion interaction between two collinear insulating wires. Using Eqs. (6)-(8), we first calculated numerically and tabulated the dispersion interaction energy $E_{v d W}(\ell, d)$ of short metallic wires of length $L=\ell A$, with separation $D=d A$ between the ends. [For fixed $\ell \ll d$, we found $E_{v d W}(\ell, d)$ to be of the conventional form $-C(\ell) d^{-6}$ as expected; see Sec. IV]. We then modeled a long insulating wire of length $L=i \ell A(i=1,2,3, \ldots)$ by a collection of short wire segments of equal length $\ell A$, placed end to end, but with electrons not allowed to flow from segment to segment. This crudely represents individual atoms or bonds on an insulating wire, as electron movement along the wire is restricted, now being confined to within each wire segment. Assuming that the mutual polarization between any two wire segments is not affected by any other wire segment composing the collinear wire system, the pretabulated dispersion interaction $E_{v d W}(\ell, d=q \ell)$ can be used between any two segments of length $\ell A$ lying in opposite wires and separated by distance $q \ell$. Summation of the dispersion interaction between each segment on one wire and all segments on the opposite wire (as illustrated in Fig. 2) then gives the total vdW interaction for insulating wires, separated by distance $D=p \ell$, in the pairwise summation approach:

$$
E_{v d W}^{\text {pairwise }}(D=p \ell)=\sum_{m, n=0}^{i-1} E_{v d W}(\ell, d=(p+m+n) \ell) .
$$

Here, each wire has length $L=i \ell$, where $i$ is an integer.

This method provides an increasingly accurate representation of two collinear wire insulators with decreasing length of the individual wire segments, as electron movement is restricted to the individual wire pieces.

(B) We also explored a more conventional approach to model an insulator by adding a harmonic pinning force $-M \Omega_{p i n}^{2} R(X)$ to the right-hand side of the equation of motion (3) for $M \ddot{R}$. This, plus transposition and inclusion of the 
pressure term (8), resulted in an eigenvalue problem of the form [cf. Eq. (6)]

$$
\begin{aligned}
\frac{M\left(\Omega^{2}-\Omega_{p i n}^{2}\right)}{n_{0} e^{2}} R(X)= & \int_{D / 2}^{L+D / 2} R\left(X^{\prime}\right)\left(\frac{-2\left(X-X^{\prime}\right)^{2}+A^{2}}{\left[\left(X-X^{\prime}\right)^{2}+A^{2}\right]^{5 / 2}}\right. \\
& \left. \pm \frac{-2\left(X+X^{\prime}\right)^{2}+A^{2}}{\left[\left(X+X^{\prime}\right)^{2}+A^{2}\right]^{5 / 2}}\right) d X^{\prime}-\frac{M B^{2}}{n_{0} e^{2}} \frac{d^{2} R}{d X^{2}}
\end{aligned}
$$

Each eigenfrequency of Eq. (10) is of the form

$$
\Omega_{i}=\sqrt{\bar{\Omega}_{i}^{2}+\Omega_{p i n}^{2}},
$$

where $\bar{\Omega}_{i}$ is an eigenfrequency of Eq. (10) with the pinning term absent. A semiconducting or insulating wire with an electronic energy gap $E_{g}$ can be modeled by including a finite pinning frequency $\Omega_{\text {pin }} \neq 0$. A metallic wire is modeled by setting $\Omega_{p i n}=0$. The idea is that each electron experiences a force tending to tie it to its labeled equilibrium position $X$. In this simple approach, the pinning energy $\hbar \Omega_{\text {pin }}$ is loosely associated with the electronic gap $E_{g}$ of an insulator, $\hbar \Omega_{\text {pin }} \approx E_{g}$. The pinning has the effect of reducing the polarizability of the electron gas, stiffening the plasmon modes and making them less affected by coupling between the wires. The modified frequencies [Eq. (11)] are then substituted into Eq. (7) to obtain the vdW energy as before. A similar pinning approach gives the conventional power laws for the vdW attraction between insulating two-dimensional sheets $\left(E_{v d W^{\propto}} \propto D^{-4}\right)$ and for distant parallel insulating wires $^{42}\left(E_{v d W^{\alpha}} \propto-D^{-5}\right)$. As we will show in Sec. IV, for the present problem in the appropriate parameter regime, the pinned approach also produced results very similar to approach (A) in which the wire was cut into short segments. Both will be seen to give results for the vdW interaction between collinear wires that is very different from the case of metallic wires.

\section{NUMERICS}

To solve Eq. (10) numerically, the integral is discretized by application of the trapezoidal rule, which, along with a zero boundary condition for the displacement at the edges of each wire, converts the integral equation (10) to a symmetric matrix eigenvalue equation of dimension $N-1$, where $N$ is the total number of partitions of the integral term in Eq. (10). With inclusion of a pinning force, the matrix eigenvalue problem is of the form

$$
\frac{M\left(\Omega^{2}-\Omega_{p i n}^{2}\right)}{n_{0} e^{2}} R_{i}=\sum_{m=1}^{N-1}\left(G_{i m} W_{m}-\frac{M B^{2} N^{2}}{L^{2} n_{0} e^{2}} P_{i m}\right) R_{m}
$$

for $1 \leqslant i \leqslant N-1$. Here, $G_{i m}$ is the matrix

$$
G_{i m}=\frac{A^{2}-2[(i-m) L / N]^{2}}{\left\{[(i-m) L / N]^{2}+A^{2}\right\}^{5 / 2}} \pm \frac{A^{2}-2[D+(i+m) L / N]^{2}}{\left\{[D+(i+m) L / N]^{2}+A^{2}\right\}^{5 / 2}} .
$$

Here, $W_{m}=L / N, 1 \leqslant m \leqslant N-1$, is the weighting function for trapezoidal integration: The end weights $W_{0}=W_{N}=L / 2 N$ are not sampled because of the pinning condition $R_{0}=R_{N}=0$, so that the $(N-1) \times(N-1)$ matrix equation (12) is symmetric.

In Eq. (12),

$$
P_{i m}=\delta_{i-1, m}-2 \delta_{i, m}+\delta_{i+1, m}
$$

is a discrete dimensionless version of the second-derivative operator. In obtaining Eq. (12) from Eq. (10), $X$ and $X^{\prime}$ have been replaced by discrete positions $X_{i}$ and $X_{m}$ defined in terms of the absolute wire length $L$, wire separation distance $D$, and $N$ by $X_{i}=D / 2+i L / N$ for $i=0,1,2, \ldots, N$ and $X_{m}=D / 2+m L / N$ for $m=0,1,2, \ldots, N$.

\section{A. Dimensionless form of equations}

The eigenvalue problem can be written in dimensionless form as follows. First, we scale all lengths by the effective radius $A$, introducing dimensionless quantities $d, \ell, r_{i}$, and $w_{i}$ such that $D=d A, L=\ell A, R_{i}=r_{i} A$, and $W_{i}=w_{i} A$. We also define $J_{i m}=A^{3} G_{i m}$ which is dimensionless. We introduce a characteristic frequency $\Omega_{0}$ defined by

$$
\Omega_{0}=\sqrt{\frac{n_{0} e^{2}}{M A^{2}}},
$$

which is $\frac{1}{2} \Omega_{P}$, where $\Omega_{P}=\sqrt{4 \pi n_{0}\left(\pi A^{2}\right)^{-1} e^{2} / M}$ is the bulk plasma frequency calculated assuming a bulk electron density $n_{3 \mathrm{D}}=n_{0}\left(\pi A^{2}\right)^{-1}$, as though the $1 \mathrm{D}$ density $n_{0}$ were distributed uniformly throughout a cylinder of radius $A$. Then, the equation of motion (12) can be written in dimensionless form as

$$
\frac{\Omega^{2}-\Omega_{p i n}^{2}}{\Omega_{0}^{2}} r_{i}=\sum_{m=1}^{N-1}\left(J_{i m} w_{m}-\beta^{2} \frac{N^{2}}{\ell^{2}} P_{i m}\right) r_{m},
$$

where

$$
J_{i m}=\frac{1-2 \ell^{2}(i-m)^{2} N^{-2}}{\left[\ell^{2}(i-m)^{2} N^{-2}+1\right]^{5 / 2}} \pm \frac{1-2\left[d+\ell(i-m) N^{-1}\right]^{2}}{\left\{\left[d+\ell(i-m) N^{-1}\right]^{2}+1\right\}^{5 / 2}}
$$

for even (+) and odd (-) solutions, respectively. In Eq. (15),

$$
\beta^{2}=\frac{B^{2}}{A^{2} \Omega_{0}^{2}}
$$

is a dimensionless measure of the importance of the pressure term. Equation (15) holds for $1 \leqslant i \leqslant N-1$.

The discrete eigenfrequencies $\Omega_{j}$ from numerical solution of the $(N-1) \times(N-1)$ matrix equation (15) were used as follows in calculating the attractive dispersion energy $E_{v d W}$ between the wires [cf. Eq. (7)]:

$$
E_{v d W}=\frac{\hbar}{2} \sum_{j=1}^{N-1}\left[\Omega_{j}^{(+)}(d)+\Omega_{j}^{(-)}(d)-2 \Omega_{j}^{(0)}(d \rightarrow \infty)\right] .
$$

Here, $\Omega_{j}^{(+)}(d)$ is the $j$ th eigenvalue of Eq. (15) with the + sign used in Eq. (16), corresponding to even plasmon modes. Similarly, $\Omega_{j}^{(-)}(d)$ is the eigenvalue with the - sign in Eq. (15), corresponding to odd modes. $\Omega_{j}^{(0)}(d \rightarrow \infty)$ is the $j$ th eigenvalue with only the first term kept in Eq. (16), corresponding to infinite separation $d \rightarrow \infty$. Note that the even and 
odd modes are degenerate in the limit $d \rightarrow \infty$, accounting for the factor of 2 in the last term of Eq. (18).

\section{B. Numerical convergence}

Numerical convergence of the dispersion interaction with respect to $N$ was also verified. A larger $N$ corresponds to dividing the integral into more partitions; consequently, as more "points" along the wire are sampled, larger $N$ means plasmon modes with shorter wavelengths are also sampled. The lowest plasmon mode wavelengths are expected to contribute negligibly to the dispersion interaction, so for sufficiently large $N$, numerical convergence of the vdW interaction should be evident. We found that $N$ up to 800 was sufficient for convergence of the dispersion interaction for wires separated by $D=2 A$ at all of the lengths treated here (up to $80 A$ ). Increasing the internal pressure, corresponding to using a larger Fermi velocity, gives a more rapid convergence of the dispersion interaction with respect to $N$ and a weaker vdW attraction between the two wires. This is because the pressure term stiffens the short-wavelength modes, making them less sensitive to the weak interaction between the wires. We also found that inclusion of the pressure improved the numerical stability.

The value of $N$ necessary for convergence (to a given fractional level) increases with wire length and also with wire separation distance. For comparison of the dispersion energy for conducting and insulating wires, and in investigating variation of separation distance for wires much longer than the separation distance, we employed $N$ sufficiently large to achieve convergence of the $\mathrm{vdW}$ interaction to about three significant figures.

\section{Choice of numerical parameters for carbon nanotubes}

The basic approach described above should apply to any system where there is an essentially $1 \mathrm{D}$ electron gas with long mean free path, such as a metallic nanowire or the conduction band of a metallic $(n, n)$ carbon nanotube. The results using Eq. (15) are dimensionless and therefore universal in a sense. However, the dispersion energy from Eq. (7) is thereby obtained in units of $\hbar \Omega_{0}$ defined in Eq. (14), and this quantity will be different for different $1 \mathrm{D}$ systems. Our dimensionless lengths are defined in terms of the Coulomb smearing length $A$, which is taken to be the radius of the quasi-1D electron gas, e.g., the tube radius in the nanotube case. It is also necessary to choose the dimensionless input parameters $\beta, \omega_{\text {pin }} \equiv \Omega_{\text {pin }} / \Omega_{0}$, to suit the particular system.

For the numerical work below, we chose parameters roughly appropriate to the conduction band of a $(5,5)$ singlewalled carbon nanotube. While a metallic nanotube is an excellent metal, it is not a free-electron metal. We therefore first establish a correspondence between our classical Newton II approach and the relevant $q \rightarrow 0$ quantal response by considering an infinitely long wire as follows. A simple model of the rigid time-oscillatory displacement of the $k$-space Bloch electron distribution under an external electric field $E_{0} \exp (i q z-i \omega t)$ gives the bare density-density response for $q \rightarrow 0$ as

$$
\chi^{0}(q, \omega)=v_{F} q^{2} /\left(\pi \omega^{2} \hbar\right) .
$$

Here, the Fermi velocity $v_{F}=\hbar^{-1} \partial \varepsilon(k) /\left.d k\right|_{k=k_{F}}$ is the gradient of the 1D Bloch energy $\varepsilon(k)$ at the Fermi point $k=k_{F} \cdot v_{F}$ can be calculated by differentiation of the analytic Bloch electron dispersion for single-walled $(n, n)$ nanotubes given in Eq. (4.6) of Saito et $a l .{ }^{43}$ In this way, we obtained the Fermi velocity of the conduction bands (those with $q=n$ and $q=2 n$ in the notation of Saito et al.) as $v_{F}=\sqrt{3} a|t| /(2 \hbar)$. Here, $|t|=3.03 \mathrm{eV}$ is the hopping parameter and $a=4.65 \mathrm{bohr}$ radii is the length of the primitive translation vector of the parent two-dimensional graphene lattice. This gives $v_{F}=9.9 \times 10^{5} \mathrm{~m} / \mathrm{s}$ independent of $n$, a value quite comparable to Fermi velocities in three-dimensional metals.

The bare response of the infinite wire can also be calculated via the Newton II approach (used in our numerical treatment outlined above for the case of finite length), giving

$$
\chi_{0}(q, \omega)=n_{0} q^{2} /\left(m \omega^{2}\right),
$$

where $n_{0}$ is the $1 \mathrm{D}$ density of the electron gas and $m$ is the effective classical mass.

By comparing Eqs. (20) and (19), we establish the equivalence

$$
n_{0} / m=v_{F} /(\pi \hbar) .
$$

Thus, the energy $\hbar \Omega_{0}$ that scales our vdW energy predictions [see Eqs. (7), (14), and (15)] is

$$
\hbar \Omega_{0}=\hbar \sqrt{\frac{v_{F}}{\pi \hbar} \frac{e^{2}}{A^{2}}}=\frac{8.02}{n} \mathrm{eV},
$$

where we have used the result ${ }^{43} A=\sqrt{3} n a /(2 \pi)$ for the radius of a $(n, n)$ carbon nanotube.

Assuming that the characteristic velocity $B$ entering the pressure term Eq. (8) is of order $v_{F}$, we find that the dimensionless pressure parameter $\beta$ appearing in Eq. (15) is of order unity. We used the value $\beta=1.38$ throughout.

We also need to choose values for the pinning frequency $\Omega_{p i n}$ that mimic the effect of an insulating energy gap. Semiconducting carbon nanotubes have electron energy gaps up to $O(1 \mathrm{eV})$. Assuming that $\hbar \Omega_{\text {pin }}$ is of this order, we obtain from the numbers above a ratio $\omega_{\text {pin }} \equiv \Omega_{p i n} / \Omega_{0}=O(n / 8)$ for a $(n, n)$ nanotube. We explored cases with values of $\omega_{\text {pin }}$ varying from 0 (representing the conduction electrons of a metallic wire) to $\omega_{\text {pin }}=1.73$ (representing strongly semiconducting electron bands).

\section{RESULTS AND DISCUSSION}

We first verified that, in the pointing geometry of interest here, our numerical coupled-plasmon approach yields the expected dispersion interaction of form $E_{v d W} \sim-C_{6}(\ell) d^{-6}$ for two wires whose (equal) dimensionless length $\ell$ is much less than their dimensionless separation $d$ measured between their ends. In this limit, the $d^{-6}$ law can be predicted analytically by modeling each piece of wire as a polarizable dipole. ${ }^{1,13}$ To test this power law explicitly, in Fig. 3, we plot $d_{c m}^{6} E_{v d W}$ vs $d$, where $d_{c m} \equiv d+\ell$ is the distance between the centers of the wires. 


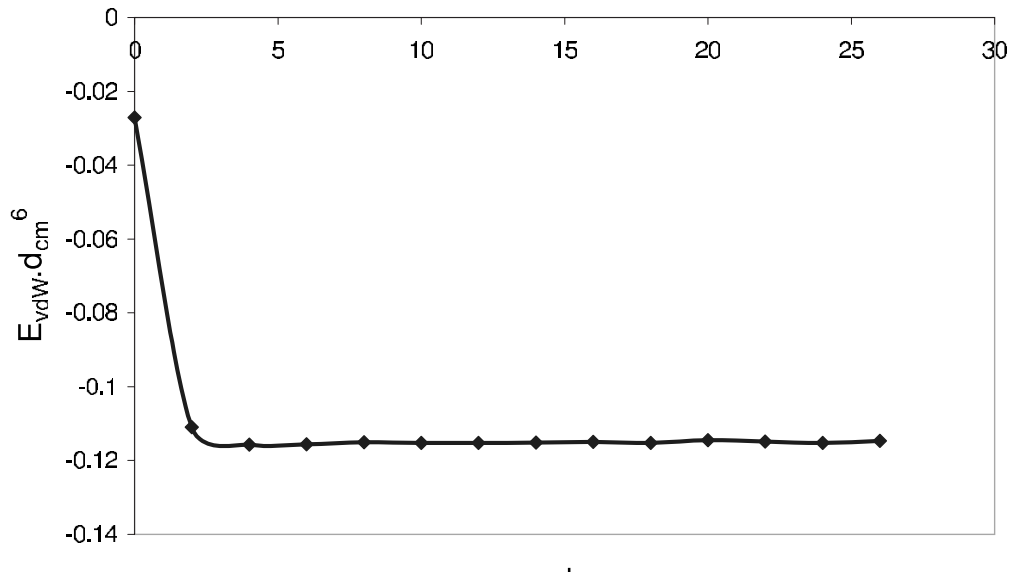

FIG. 3. Dispersion energy $d_{c m}^{6} E^{v d W}$ vs $d$ for dimensionless length $\ell=2$, pressure parameter $\beta=1.38$, and zero pinning.
For each chosen value of $\ell$, the plot settles down to a constant value $-C_{6}(\ell)$ for $d \gg \ell$, which confirms the expectation. This basic $d^{-6}$ behavior is observed both for metallic wires and for semiconducting wires (modeled by adding a pinning force as described above). For a given wire length $\ell$, the coefficient $C_{6}(\ell)$ depends considerably on the pinning frequency $\omega_{\text {pin }}$, i.e., metallic wires $\left(\omega_{\text {pin }}=0\right)$ attract more strongly than insulating ones with the same concentration of vdW interacting electrons, but still with a $d^{-6}$ law for the energy.

Much more interesting is the behavior of the dispersion energy when the separation $d$ is less than the length $\ell$. Here, a single dipole does not adequately represent the electronic response of a wire, and consequently no single power law $d^{p}$ emerges for the energy $E_{v d W}(d)$ because a multipolar expansion would be required in order to generalize the dipolar argument. We are therefore out of the "asymptotic" region of a distant vdW interaction, though still not in a region of electronic cloud overlap. In this regime, a conventional approach would be to sum (integrate) contributions of form $-C\left|r_{1}-r_{2}\right|^{-6}$ from small segments of the two wires at positions $r_{1}$ and $r_{2}$, respectively. This approach predicts that, for fixed $d$, the dispersion energy for increasing wire length $\ell$ will saturate rapidly as soon as $\ell$ exceeds $d$, to a value proportional to $-d^{-4}$. We find that this saturation does occur for insulating wires, as modeled either with a substantial pinning frequency $\omega_{\text {pin }}$ or by cutting a metallic wire into mutually insulated pieces shorter than the separation. It is definitely not true for intact metallic wires $\left(\omega_{\text {pin }}=0\right)$ as modeled by our full numerical coupled-plasmon approach. In the metallic case, the interaction continues to grow with wire length and still has not saturated for wires many times longer than the separation. These findings are illustrated in Fig. 4.

We also investigated the behavior of our coupled-plasmon interaction right down to zero separation of the wires, see Fig. 5. Of course, a realistic treatment of this regime would require the inclusion of covalent and other forces that are not described in our formalism and that are specific to the particular quasi-1D system. Furthermore, a detailed plasmon treatment even of the dispersion part of the energy in this regime would require electronic response functions beyond the long-wavelength description that we have used. However, our results establish the important result that our method saturates naturally to a finite value at contact, unlike empirical vdW correction schemes of the $\Sigma C_{i j} R_{i j}^{-6}$ type,

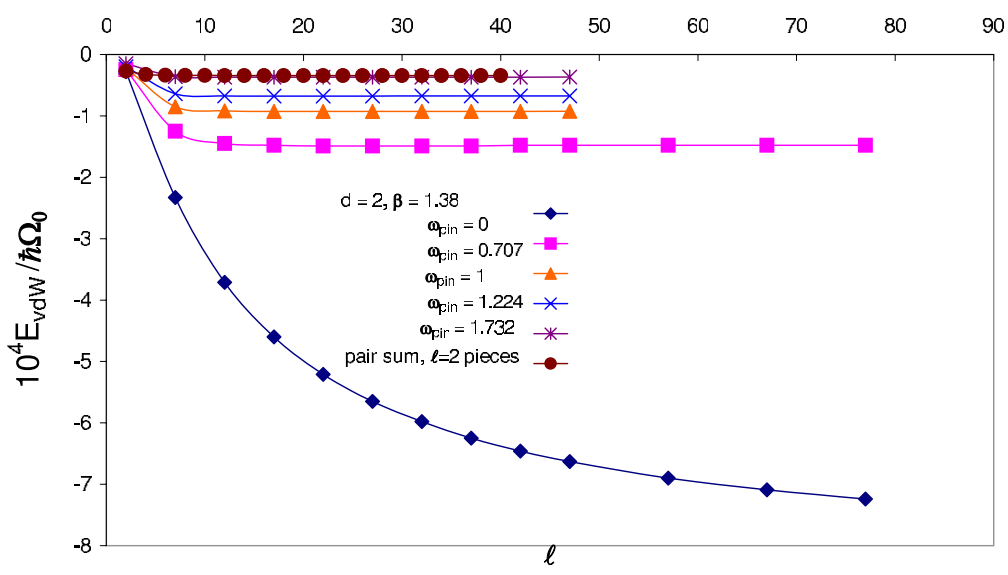

FIG. 4. (Color online) Dispersion energy $E_{v d W}$ vs wire length $\ell$ for fixed separation $d=2$ and pressure parameter $\beta=1.38$. Diamonds: metallic wire $\left(\omega_{\text {pin }}=0\right)$. Solid squares: semiconducting wire with $\omega_{\text {pin }}=0.707$. Solid triangles: semiconducting wire with $\omega_{\text {pin }}=1.0$. Crosses: semiconducting wire with $\omega_{\text {pin }}=1.224$. Asterisks: semiconducting wire with $\omega_{\text {pin }}=1.732$. Solid circles: metallic wire $\left(\omega_{\text {pin }}=0\right)$ that has been cut into pieces of length 2 . The unit of energy (on the vertical axis) is $\hbar \Omega_{0}$ as defined in Eqs. (14) and (21). For the conduction band of a $(5,5)$ carbon nanotube, this energy unit is $1.6 \mathrm{eV}$. 


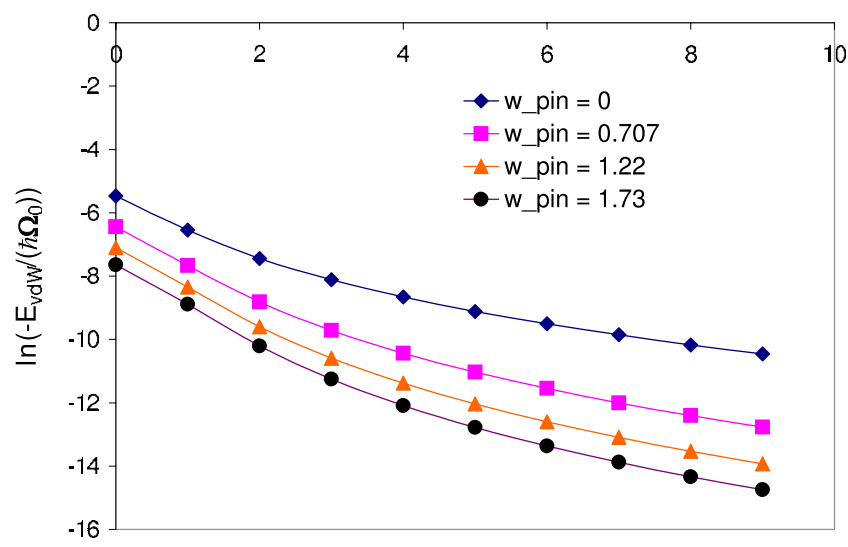

d

FIG. 5. (Color online) Coupled-plasmon interaction at small wire separation $d$.

which have to be cut off in a somewhat arbitrary manner to avoid divergence. Another significant conclusion from our numbers in this small-separation regime concerns the relative strength of the dispersion energy for metallic and nonmetallic cases. Because the analytic results in various geometries $^{12,26}$ for the metallic interaction fall off with a lower power and hence dominate the insulating result at large distance, one might speculate that the opposite ordering holds at small separation, i.e., that the insulating interaction is stronger than the metallic one at small separation. However, such an argument depends on the assumption of the same single power law for small separations as well as large. This is not the case, and at small separations $d$, a power series rather than a single power would be required for this type of analysis. In fact, our results show that the metallic attraction is stronger than the insulating one at all separations for constant electron number density.

\section{SUMMARY AND CONCLUSIONS}

We have explored the dispersion interaction between two collinear 1D electronic systems (wires) separated by a vacuum gap in the electromagnetically nonretarded regime. We considered the metallic case and also two models of an insulating and/or semiconducting case. The metallic case was modeled via free 1D electrons with an electronic pressure term, but this model was also matched to a quantum band model for the electrons for the purpose of modeling the conduction electron bands in carbon nanotubes. For the insulating cases, either a harmonic pinning force was added to the equations or a model of very short, mutually insulated metallic pieces was used, with rather similar results to the case of a pinning force. Our calculations provide significant insight additional to that previously obtained from asymptotic dispersion energy calculations ${ }^{12,13,26,27,29}$ on low-dimensional systems with at least one infinite spatial dimension. These previous analytic calculations were all performed for the asymptotic regime where a single power law (sometimes multiplied by a logarithm) suffices to describe the dispersion energy as a function of separation $D$. These previous works showed that the distant interaction in these highly anisotropic systems shows major qualitative differences between the metallic and insulating cases. Specifically, the power law is different in the two cases, with the metallic interaction being stronger in the asymptotic regime studied.

In contrast to those fully asymptotic calculations, we treated wires of finite length $L$, with a finite separation $D$ between their ends, a regime where a single power law in $D$ does not describe the interaction. Unlike the asymptotic cases, we find that the interaction at finite separations is a continuous function of the pinning frequency (i.e., a continuous function of the electronic energy gap). Our coupledplasmon dispersion energy does, however, remain a strong function of the gap right down to the limit of contacting wires, where it remains finite, unlike asymptotic formulas for the same quantity. In particular, we find a large difference between the attractive dispersion energy of the most polarizable electron bands of metallic and semiconducting carbon nanotubes. The dispersion energy was found to decrease monotonically with energy gap at all values of $L$ and $D$ that we studied.

We also found that a conventional pairwise summation of terms of form $-C R^{-6}$ is not an adequate description of metallic wires, with a major discrepancy between this model and our coupled-plasmon results. Specifically, the coupledplasmon energy, while similar to the summation approach for very short wires, continues to grow with length $L$ at fixed separation $D$, while the $-\Sigma C R^{-6}$ energy saturates rapidly to an $L \rightarrow \infty$ value that is much smaller than the coupledplasmon energy.

A more detailed microscopic model, including a detailed orbital description with Pauli repulsion and nondispersive bonding forces, will be required to describe the cohesive forces at distances where the electronic clouds overlap. The present results suggest, however, that long-wavelength coupled charge fluctuations will still enhance the vdW force at these distances, necessitating a highly nonlocal model of the Coulomb screening even in this limit. Theories that yield a $\Sigma R^{-6}$ asymptotics cannot be relied upon to achieve this.

\footnotetext{
*angela.white@anu.edu.au

†j.dobson@griffith.edu.au

${ }^{1}$ J. F. Dobson, K. McLennan, A. Rubio, J. Wang, T. Gould, and B.

P. Dinte, Aust. J. Chem. 54, 513 (2001).

${ }^{2}$ I. N. Berezovsky and E. N. Trifonov, J. Mol. Biol. 307, 1419
}

(2001).

${ }^{3}$ A. G. Street and S. L. Mayo, Proc. Natl. Acad. Sci. U.S.A. 96, 9074 (1999).

${ }^{4}$ P. P. Priavalov, J. Chem. Thermodyn. 29, 447 (1997).

${ }^{5}$ K. Autumn, Y. Lian, S. T. Hsieh, W. Zesch, W. P. Chan, T. W. 
Kenny, R. Fearing, and R. J. Full, Nature (London) 405, 681 (2000).

${ }^{6}$ K. Autumn, M. Sitti, Y. Lian, A. M. Peattie, W. R. Hansen, S. Sponberg, T. W. Kenny, R. Fearing, J. N. Israelachvili, and R. J. Full, Proc. Natl. Acad. Sci. U.S.A. 99, 12252 (2002).

${ }^{7}$ A. Rubio, J. L. Corkill, and M. L. Cohen, Phys. Rev. B 49, 5081 (1994).

${ }^{8}$ A. G. Marinopoulos, L. Wirtz, A. Marini, V. Olevano, A. Rubio, and L. Reining, Appl. Phys. A: Mater. Sci. Process. 78, 1157 (2004).

${ }^{9}$ J. Bernholc, D. Brenner, M. B. Nardelli, V. Meunier, and C. Roland, Annu. Rev. Mater. Res. 32, 347 (2002).

${ }^{10}$ C. Dekker, Phys. Today 52 (5), 22 (1999).

${ }^{11}$ J. F. Dobson and A. Rubio, arXiv:cond-mat/0502422 (unpublished).

${ }^{12}$ J. F. Dobson, A. White, and A. Rubio, Phys. Rev. Lett. 96, 073201 (2006).

${ }^{13}$ J. F. Dobson, J. Wang, B. P. Dinte, K. McLennan, and H. M. Lee., Int. J. Quantum Chem. 101, 570 (2005).

${ }^{14}$ J. Mahanty and B. Ninhan, Dispersion Forces (Academic, London, 1976).

${ }^{15}$ E. M. Lifshitz, Sov. Phys. JETP 2, 73 (1956).

${ }^{16}$ I. E. Dzyaloshinskii, E. M. Lifshitz, and L. P. Pitaevskii, Adv. Phys. 10, 165 (1961).

${ }^{17}$ M. Dion, H. Rydberg, E. Schröder, D. C. Langreth, and B. I. Lundqvist, Phys. Rev. Lett. 92, 246401 (2004).

${ }^{18}$ H. Rydberg, N. Jacobson, P. Hyldgaard, S. I. Simak, B. I. Lundqvist, and D. C. Langreth, Surf. Sci. 532, 606 (2003).

${ }^{19}$ H. Rydberg, M. Dion, N. Jacobson, E. Schröder, P. Hyldgaard, S. I. Simak, D. C. Langreth, and B. I. Lundqvist, Phys. Rev. Lett. 91, 126402 (2003).

${ }^{20}$ E. Hult, Y. Andersson, B. I. Lundqvist, and D. C. Langreth, Phys. Rev. Lett. 77, 2029 (1996)

${ }^{21}$ H. Rydberg, B. I. Lundqvist, D. C. Langreth, and M. Dion, Phys.
Rev. B 62, 6997 (2000).

${ }^{22}$ E. Schröder and P. Hyldgaard, Surf. Sci. 532, 880 (2003).

${ }^{23}$ A. Pudzer, M. Dion, and D. C. Langreth, J. Chem. Phys. 124, 164105 (2006).

${ }^{24}$ S. D. Chakarova-Käck, E. Schröder, B. I. Lundqvist, and D. C. Langreth, Phys. Rev. Lett. 96, 146107 (2006).

${ }^{25}$ D. C. Langreth, M. Dion, H. Rydberg, E. Schröder, P. Hyldgaard, and B. I. Lundqvist, Int. J. Quantum Chem. 101, 599 (2005).

${ }^{26}$ D. B. Chang, R. L. Cooper, J. E. Drummond, and A. C. Young, Phys. Lett. 37A, 311 (1971); we thank N. Drummond for alerting us to this work.

${ }^{27}$ B. E. Sernelius and P. Björk, Phys. Rev. B 57, 6592 (1998).

${ }^{28}$ J. F. Dobson and J. Wang, Phys. Rev. Lett. 82, 2123 (1999).

${ }^{29}$ M. Böstrom and B. E. Sernelius, Phys. Rev. B 61, 2204 (2000).

${ }^{30}$ J. F. Dobson and A. White (unpublished).

${ }^{31}$ A. White, Honours thesis, Griffith University, 2003.

${ }^{32}$ A. D. Grozav and E. Condrea, J. Phys.: Condens. Matter 16, 6507 (2004)

${ }^{33}$ R. Saito, G. Dresselhaus, and M. Dresselhaus, Physical Properties of Carbon Nanotubes (Imperial College Press, London, 1998).

${ }^{34}$ D. L. Blundell, Ph.D. thesis, University of Wollongong, 2005.

${ }^{35}$ S. V. Rotkin and K. Hess, J. Comput. Electron. 1, 323 (2002).

${ }^{36}$ F. Forstmann and H. Stenschke, Phys. Rev. B 17, 1489 (1978).

${ }^{37}$ A. Eguiluz, Phys. Rev. B 19, 1689 (1979).

${ }^{38}$ J. F. Dobson and H. M. Le, Phys. Rev. B 66, 075301 (2002).

${ }^{39}$ S. DasSarma and J. J. Quinn, Phys. Rev. B 20, 4872 (1979).

${ }^{40}$ A. J. Bennett, Phys. Rev. B 1, 203 (1970).

${ }^{41}$ G. Barton, Rep. Prog. Phys. 42, 963 (1979).

${ }^{42}$ J. F. Dobson, Surf. Sci. 601, 5667 (2007).

${ }^{43}$ R. Saito, G. Dresselhaus, and M. S. Dresselhaus, Physical Properties of Carbon Nanotubes (Imperial College Press, London, 1998). 